\title{
The Responses of Tissues from the Brain, Heart, Kidney, and Liver to Resuscitation following Prolonged Cardiac Arrest by Examining Mitochondrial Respiration in Rats
}

\author{
Junhwan Kim, ${ }^{1}$ José Paul Perales Villarroel, ${ }^{1}$ Wei Zhang, ${ }^{1}$ Tai Yin, ${ }^{1}$ Koichiro Shinozaki, ${ }^{1}$ \\ Angela Hong, ${ }^{2}$ Joshua W. Lampe, $^{1}$ and Lance B. Becker ${ }^{1}$ \\ ${ }^{1}$ Center for Resuscitation Science, Department of Emergency Medicine, University of Pennsylvania, Philadelphia, PA 19104, USA \\ ${ }^{2}$ College of Liberal Arts and Sciences, Villanova University, Villanova, PA 19085, USA
}

Correspondence should be addressed to Junhwan Kim; junhwan.kim@uphs.upenn.edu

Received 30 June 2015; Revised 2 September 2015; Accepted 3 September 2015

Academic Editor: Massimo Collino

Copyright (c) 2016 Junhwan Kim et al. This is an open access article distributed under the Creative Commons Attribution License, which permits unrestricted use, distribution, and reproduction in any medium, provided the original work is properly cited.

Cardiac arrest induces whole-body ischemia, which causes damage to multiple organs. Understanding how each organ responds to ischemia/reperfusion is important to develop better resuscitation strategies. Because direct measurement of organ function is not practicable in most animal models, we attempt to use mitochondrial respiration to test efficacy of resuscitation on the brain, heart, kidney, and liver following prolonged cardiac arrest. Male Sprague-Dawley rats are subjected to asphyxia-induced cardiac arrest for $30 \mathrm{~min}$ or $45 \mathrm{~min}$, or $30 \mathrm{~min}$ cardiac arrest followed by $60 \mathrm{~min}$ cardiopulmonary bypass resuscitation. Mitochondria are isolated from brain, heart, kidney, and liver tissues and examined for respiration activity. Following cardiac arrest, a timedependent decrease in state- 3 respiration is observed in mitochondria from all four tissues. Following 60 min resuscitation, the respiration activity of brain mitochondria varies greatly in different animals. The activity after resuscitation remains the same in heart mitochondria and significantly increases in kidney and liver mitochondria. The result shows that inhibition of state-3 respiration is a good marker to evaluate the efficacy of resuscitation for each organ. The resulting state-3 respiration of brain and heart mitochondria following resuscitation reenforces the need for developing better strategies to resuscitate these critical organs following prolonged cardiac arrest.

\section{Introduction}

Cardiac arrest (CA) induces whole-body ischemia, which causes damage to multiple organs, including the brain, heart, kidney, and liver [1]. The damage to these vital organs is responsible for the high mortality and morbidity of patients with CA. However, how each organ responds to ischemia/reperfusion and further contributes to the mortality of patients is not understood. This information is critical to develop better resuscitation strategies to enhance the survival rate of patients, which has not improved over the last few decades.

The exact pathology of ischemia/reperfusion injury remains poorly described in CA. In particular, the fact that additional damage following resuscitation, when observed, is from reperfusion injury or poor perfusion is not clear
[2]. The interpretation is more complicated by the fact that damage to one tissue can influence the severity of damage or recovery of other tissues [3]. This complication may be overcome by monitoring the function of each organ following ischemia and resuscitation. However, direct measurement of organ function is not practicable in most animal models of CA.

Ischemia causes various alterations in the mitochondrial electron transport chain $[4,5]$. Particularly, respirational defects are the most commonly observed ischemic alteration $[6-8]$ and rescuing mitochondrial function is the key to successful resuscitation [9]. These reports suggest that mitochondrial respiration is a good indicator to examine the efficacy of resuscitation protocols on individual tissues. To further explore this hypothesis, we subjected rats to asphyxia-induced CA and cardiopulmonary bypass (CPB) 


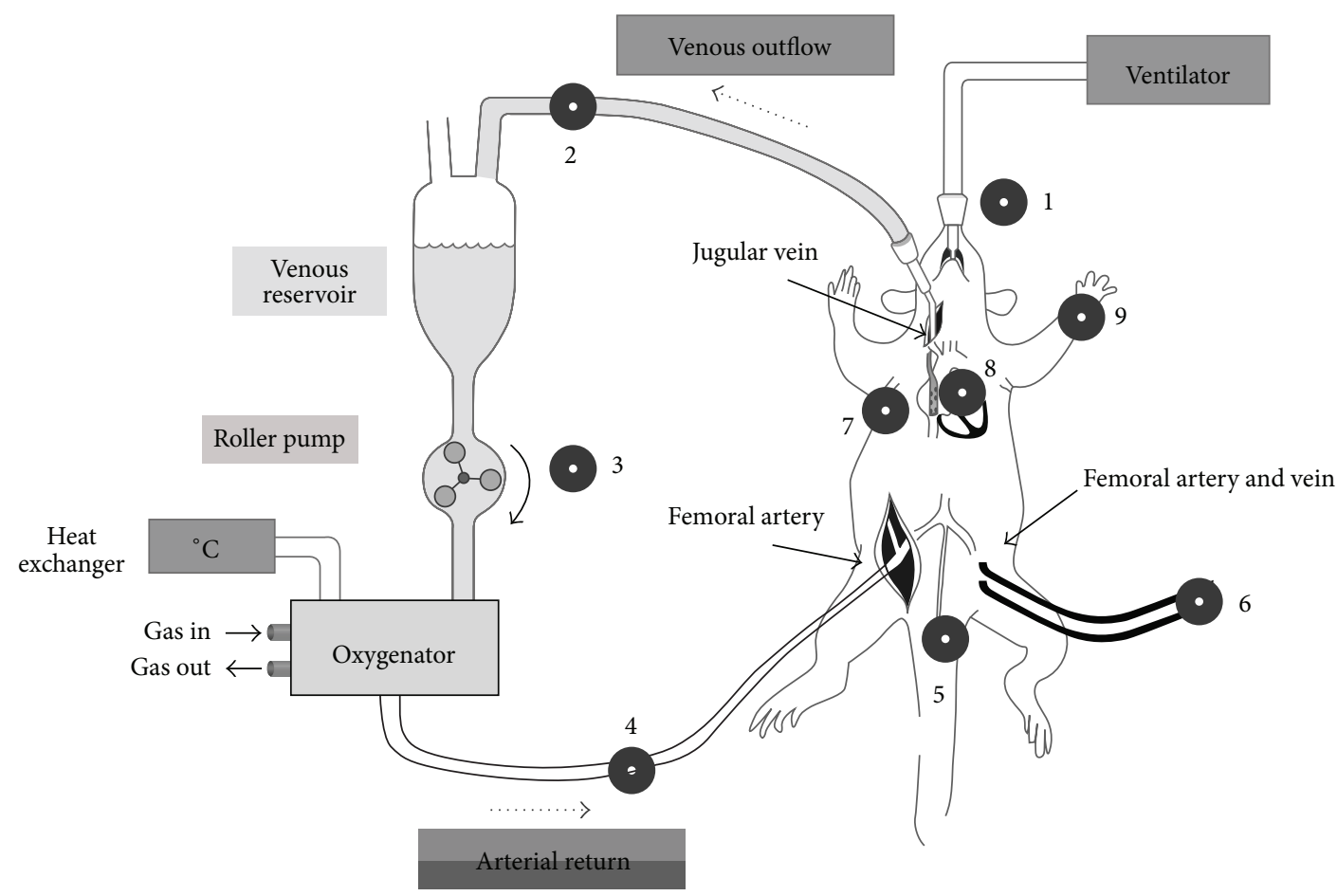

SCHEMe 1: Diagram showing surgical procedure of asphyxial CA and CPB resuscitation. 1. Capnograph. 2. Oxygen saturation and hematocrit (Critline). 3. Pump. 4. Temperature, $\mathrm{PO}_{2}$, and system pressure of arterial return. 5. Rectal temperature. 6. Arterial and central venous blood pressure, ABG. 7. ECG. 8. Esophageal temperature. 9. Pulse oximetry.

resuscitation. Mitochondria were isolated from brain, heart, kidney, and liver tissues and examined for respiration activity. The CPB system is advantageous when studying the effect of reperfusion because mechanically supported circulation provides a relatively consistent blood flow compared to conventional CPR $[10,11]$, in which the blood flow relies on the recovery of heart function.

We first compared mitochondrial state-3 (ADP-dependent), state-4 (ADP-limited), and uncoupled respiration activities between $30 \mathrm{~min}$ CA and $45 \mathrm{~min}$ CA to understand how the activities change over time under ischemic conditions. The comparison also tests whether the respiration activity observed following $30 \mathrm{~min}$ CA has reached its minimum or whether an additional decrease is possible. We then compared $30 \mathrm{~min}$ CA to $30 \mathrm{~min}$ CA followed by $60 \mathrm{~min}$ $\mathrm{CPB}$ resuscitation. The results show that state- 3 respiration is a good indicator to provide insights into tissue-specific responses of these vital organs to $\mathrm{CA}$ and resuscitation.

\section{Materials and Methods}

2.1. Animals. The experimental protocol was approved by the Institutional Animal Care and Use Committee of the University of Pennsylvania. Adult male Sprague-Dawley rats (weight $465-530 \mathrm{~g}$ ), housed in a rodent facility with unrestricted access to food and water, were used for the study. The numbers of animals were 8 for control, 7 for $30 \mathrm{~min} \mathrm{CA}, 6$ for $45 \mathrm{~min} \mathrm{CA}$, and 8 for $30 \mathrm{~min} \mathrm{CA}$ plus $60 \mathrm{~min} \mathrm{CPB}$ resuscitation. All data were presented as mean \pm standard deviation. Group comparisons were made with one-way analysis of variance and Bonferroni's test was used as a post hoc test; a $p$ value $<0.05$ was considered statistically significant.

2.2. Asphyxia-Induced Cardiac Arrest and Cardiopulmonary Bypass. The detailed procedures were published elsewhere [12] and the experimental setup of the model is shown in Scheme 1. Briefly, rats were anesthetized with $4 \%$ isoflurane and ventilated with an orotracheally intubated catheter with $2 \%$ isoflurane. Heparin (150 U) and vecuronium (1 mg) were administered through the left femoral vein. Asphyxia was induced by stopping the ventilator and isoflurane was discontinued thereafter. Within $3 \mathrm{~min}$ of asphyxia, mean arterial pressure fell below $20 \mathrm{mmHg}$, our definition of CA [12]. Following $30 \mathrm{~min} \mathrm{CA}$, resuscitation was started with the initiation of $\mathrm{CPB}$ flow and resumption of ventilation. The $\mathrm{CPB}$ flow rate was adjusted to meet venous outflow. At the end of experiment, rats were sacrificed by decapitation to collect the tissues. Control rats were decapitated $7 \mathrm{~min}$ after administration of isoflurane.

\subsection{Mitochondria Isolation}

2.3.1. General Procedure. All procedures were performed at $4^{\circ} \mathrm{C}$. The mitochondria isolation protocol was developed by modification of the method used for muscle mitochondria [13]. Each brain, heart, liver, and kidney was immediately placed in mitochondrial isolation buffer (MESH) composed 
of $210 \mathrm{mM}$ mannitol, $70 \mathrm{mM}$ sucrose, $10 \mathrm{mM}$ Hepes, and $0.2 \mathrm{mM}$ EGTA, at $\mathrm{pH}$ 7.3. The tissues were trimmed in MESH buffer to remove spinal cord, extraventricular tissue, and fats, blot-dried on filter paper, weighed, and placed in MESH buffer freshly supplemented with $0.2 \% \mathrm{w} / \mathrm{v}$ fatty acidfree BSA. The tissues were homogenized with a teflon/glass motor-driven homogenizer (Glas-Col LLC., Terre Haute, IN). A Beckman model J-30-1 centrifuge (JA-30.50 rotor) was used with low speed centrifugation at $5600 \times \mathrm{g}$ for $1 \mathrm{~min}$ and high speed centrifugation at $10000 \times \mathrm{g}$ for $6 \mathrm{~min}$. Mitochondria concentrations were determined by the BCA assay and expressed as $\mathrm{mg}$ mitochondrial protein/g tissue.

2.3.2. Brain Mitochondria Isolation. Minced brain tissue in MESH-BSA (10 mL/g tissue) was homogenized for 8 strokes at the setting of 40 . The homogenates were centrifuged at low speed and the supernatant was poured into a polycarbonate tube. The pellet was homogenized and centrifuged as above and the pooled supernatant was centrifuged at high speed. The supernatant was poured out gently until synaptosomes layer reached the top. The remaining loose pellet was suspended with $20 \mathrm{~mL}$ of $12.5 \%$ Percoll in MESH (v:v) and centrifuged at high speed. The supernatant was gently decanted with pipets without disturbing the mitochondria pellet (usually $\sim 200 \mu \mathrm{L}$ buffer remains). Finally, the pellet was resuspended in $20 \mathrm{~mL}$ of MESH buffer and centrifuged at high speed. The mitochondria pellet was suspended in $0.05 \mathrm{~mL} \mathrm{MESH}$ per $\mathrm{g}$ tissue to yield $\sim 20 \mathrm{mg} / \mathrm{mL}$ protein concentration.

2.3.3. Heart Mitochondria Isolation. Minced heart tissue in MESH-BSA ( $15 \mathrm{~mL} / \mathrm{g}$ tissue) was homogenized for 12 strokes at the setting of 30 [13]. The homogenates were centrifuged at low speed and the supernatant was saved. The homogenization of the pellet and centrifugation were repeated two more times (the third homogenization was in $10 \mathrm{~mL} / \mathrm{g}$ tissue). The pooled supernatant was centrifuged at high speed. The pellet was resuspended in $20 \mathrm{~mL}$ of MESH buffer and centrifuged at high speed again. The mitochondria pellet was suspended in $0.5 \mathrm{~mL}$ MESH per $\mathrm{g}$ tissue to yield $\sim 25 \mathrm{mg} / \mathrm{mL}$ protein concentration.

2.3.4. Kidney and Liver Mitochondria Isolation. Minced kidney or liver tissue in MESH-BSA (10 mL/g tissue) was homogenized for 3 strokes at the setting of 30 . The homogenates were centrifuged at low speed and the supernatant was centrifuged at high speed. The pellet was resuspended in $20 \mathrm{~mL}$ of MESH buffer and centrifuged at high speed. The mitochondria pellet was suspended in $0.4 \mathrm{~mL}$ MESH per g tissue to yield $30 \mathrm{mg} / \mathrm{mL}$ protein concentration for kidney and $\sim 45 \mathrm{mg} / \mathrm{mL}$ for liver mitochondria.

2.4. Oxidative Phosphorylation. Oxygen consumption was measured using a Strathkelvin oxygen electrode $\left(30^{\circ} \mathrm{C}\right)$ in a buffer containing $80 \mathrm{mM} \mathrm{KCl}, 50 \mathrm{mM}$ MOPS, $1 \mathrm{mM}$ EGTA, $5 \mathrm{mM} \mathrm{KH}_{2} \mathrm{PO}_{4}$, and $1 \mathrm{mg}$ defatted BSA/mL at $\mathrm{pH} 7.4[14,15]$. State-3, state-4, and uncoupled respirations were measured in $150 \mu \mathrm{L}$ of the mitochondrial suspension $(0.5 \mathrm{mg} / \mathrm{mL})$ using

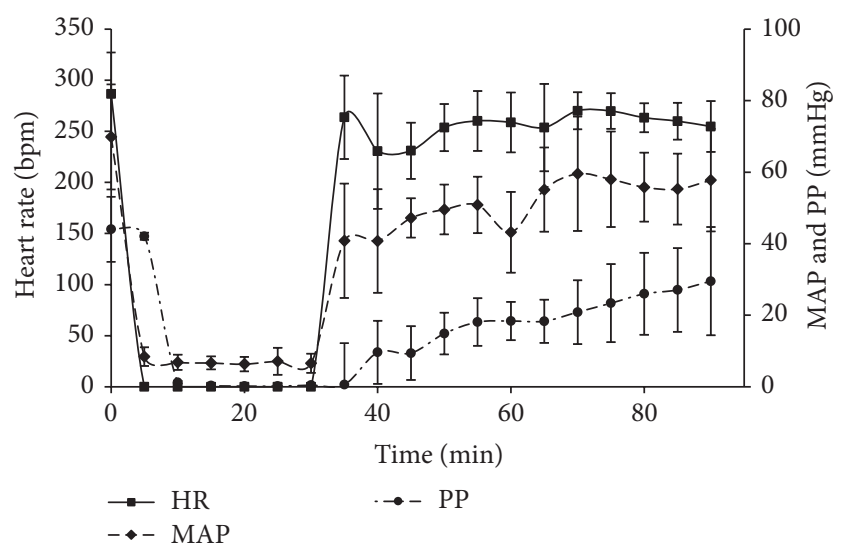

FIGURE 1: Heart rate (HR), mean arterial pressure (MAP), and pulse pressure (PP) following $30 \mathrm{mim} \mathrm{CA}$ and $60 \mathrm{~min} \mathrm{CPB}$ resuscitation.

glutamate + malate as substrates. Respiration rates were expressed as nanoatoms oxygen consumed $/ \mathrm{min} / \mathrm{mg}$ mitochondrial protein.

\section{Results}

3.1. Physiological Outcomes. Cardiac data on heart function following CA and CPB resuscitation is similar to our previously reported data (Figure 1) [16, 17]. Within $5 \mathrm{~min}$ of asphyxia, mean arterial pressure (MAP) fell below $10 \mathrm{mmHg}$ and heart rate (HR) and pulse pressure (PP) became essentially zero. Following $60 \mathrm{~min} \mathrm{CPB}$ resuscitation, HR, MRP, and $\mathrm{PP}$ reached 89,83 , and $76 \%$ of the initial rates, respectively. Heart function, however, is sustainable only with CPB support. Return of spontaneous circulation (ROSC) was achieved in $\sim 7 \mathrm{~min}$. As shown previously, rats did not show any corneal reflexes or response to toe pinching. Sporadic urination was observed during $60 \mathrm{~min} \mathrm{CPB}$ resuscitation. Based on these data, we concluded that the heart was significantly recovered with a moderate functional loss. The brain had no observable function and the kidney had adequate function following resuscitation.

3.2. Mitochondrial Isolation Yield. Mitochondria were isolated. Table 1 summarizes a complete set of data from examination of isolated mitochondria for this study. Scheme 1 and Figures 1-4 are based on Table 1 to highlight changes in mitochondrial function following extended period of ischemia or $\mathrm{CPB}$ resuscitation from $30 \mathrm{~min} \mathrm{CA}$. The isolation yields of brain and heart mitochondria were the same between the control and CA groups, suggesting no significant structural decomposition occurred until 45 min CA (Table 1). The yields for kidney and liver mitochondria were decreased following CA. The decrease was from increased tissue weight caused by increased blood content, which was clearly visible in tissue homogenates. In fact, the weight of kidney increased from $1.57 \mathrm{~g}$ (control) to $1.83 \mathrm{~g}$ (CA30) and $1.84 \mathrm{~g}$ (CA45) following $\mathrm{CA}$. The difference in the tissue weight exactly compensates the difference in isolation yield of kidney mitochondria. The decrease in the liver mitochondrial yield was also attributable to increased tissue weight, as the increased blood 
TABLE 1: Mitochondrial isolation yield ( $\mathrm{mg} / \mathrm{g}$ tissue) and respiration activities (NO/min/mg mitochondrial protein).

\begin{tabular}{|c|c|c|c|c|c|c|c|c|}
\hline & \multicolumn{4}{|c|}{ Isolation yield } & \multicolumn{4}{|c|}{ State-3 } \\
\hline & Con. & CA30 & CA45 & $\mathrm{CA} 30+\mathrm{BP} 60$ & Con. & CA30 & CA45 & $\mathrm{CA} 30+\mathrm{BP} 60$ \\
\hline Brain & $1.52 \pm 0.22$ & $1.44 \pm 0.33$ & $1.61 \pm 0.40$ & $0.99 \pm 0.41^{*}$ & $150 \pm 16.6$ & $87.9 \pm 20.9^{*}$ & $71.2 \pm 10.2^{*}$ & $87.6 \pm 37.3^{*}$ \\
\hline Heart & $20.4 \pm 1.1$ & $19.2 \pm 2.3$ & $19.9 \pm 2.0$ & $18.9 \pm 2.4$ & $178.3 \pm 18.9$ & $152.0 \pm 28.1$ & $124.0 \pm 27.2^{*}$ & $146.4 \pm 16.6^{*}$ \\
\hline Kidney & $17.8 \pm 2.8$ & $14.6 \pm 1.3^{*}$ & $15.3 \pm 1.0$ & $15.1 \pm 1.9$ & $147.7 \pm 8.7$ & $82.4 \pm 14.8^{*}$ & $54.7 \pm 15.5^{* \ddagger}$ & $103.3 \pm 7.8^{* \ddagger}$ \\
\hline \multirow[t]{3}{*}{ Liver } & $22.7 \pm 2.4$ & $16.3 \pm 1.8^{*}$ & $19.2 \pm 2.2^{*}$ & $26.6 \pm 3.4^{\ddagger}$ & $87.9 \pm 11.7$ & $65.1 \pm 4.9^{*}$ & $50.4 \pm 6.9^{* \ddagger}$ & $89.8 \pm 7.6^{\ddagger}$ \\
\hline & \multicolumn{4}{|c|}{ State-4 } & \multicolumn{4}{|c|}{ Uncoupled } \\
\hline & Con. & CA30 & CA45 & $\mathrm{CA} 30+\mathrm{BP} 60$ & Con. & CA30 & CA45 & $\mathrm{CA} 30+\mathrm{BP} 60$ \\
\hline Brain & $21.4 \pm 5.5$ & $19.6 \pm 6.0$ & $20.9 \pm 3.4$ & $18.7 \pm 4.6$ & $166.3 \pm 24.5$ & $93.0 \pm 29.2^{*}$ & $74.6 \pm 11.4^{*}$ & $101.4 \pm 54.8^{*}$ \\
\hline Heart & $21.4 \pm 5.2$ & $23.7 \pm 6.0$ & $35.8 \pm 8.3^{* \neq}$ & $19.7 \pm 4.1$ & $182.8 \pm 32.7$ & $171.7 \pm 21.6$ & $140.0 \pm 37.2$ & $144.0 \pm 17.4$ \\
\hline Kidney & $20.6 \pm 2.8$ & $16.3 \pm 4.6$ & $18.7 \pm 5.0$ & $15.4 \pm 2.7$ & $162.3 \pm 10.3$ & $91.1 \pm 24.5^{*}$ & $56.7 \pm 20.7^{* \ddagger}$ & $115.0 \pm 15.5^{*}$ \\
\hline Liver & $12.0 \pm 3.1$ & $11.9 \pm 3.8$ & $14.5 \pm 2.4$ & $12.5 \pm 3.8$ & $120.3 \pm 22.2$ & $97.9 \pm 8.6^{*}$ & $78.0 \pm 12.0^{*}$ & $127.1 \pm 9.3^{\ddagger}$ \\
\hline
\end{tabular}

*Against control; ${ }^{\ddagger}$ against CA30.

was observed in the liver homogenates as well following CA. However, brain weight was not increased following CA.

$\mathrm{CPB}$ resuscitation following $30 \mathrm{~min} \mathrm{CA}$ decreased the average brain mitochondrial isolation yield by $40 \%$ and the average had a high variability ranging from 0.3 to $1.6 \mathrm{mg}$ per $\mathrm{g}$ tissue. The isolation yield of heart mitochondria did not change following CA or resuscitation. The kidney mitochondrial yield was still as low as CA groups due to the same increased tissue weight $(1.88 \mathrm{~g})$. Interestingly, the isolation yield of liver mitochondria was increased to the level which is slightly higher than the control following resuscitation.

3.3. Mitochondrial Respiration following CA. Following CA, state-3 respiration declined proportionally to the duration of ischemia in mitochondria from all four tissues (Table 1). Compared to their control rates, state- 3 respiration activities were decreased by $41 \%$ in brain mitochondria, by $15 \%$ in heart mitochondria, by $44 \%$ in kidney mitochondria, and by $26 \%$ in liver mitochondria following $30 \mathrm{~min} \mathrm{CA}$. Following $45 \mathrm{~min}$ CA, the rates were further decreased by $53,27,63$, and $43 \%$, respectively. Uncoupled respiration mirrored state-3 respiration in all four mitochondria, suggesting that respirational defect resides in the electron transport chain system. The relative activities of state- 3 respiration of mitochondria were plotted against CA time (Figure 2). The response curves for mitochondria from all four tissues were linear with excellent $R^{2}$ values (0.983-0.998), showing that respiration is a good indicator of ischemia. The decrease rates observed in brain and kidney mitochondria were greater than those which occurred in heart and liver mitochondria. This observation is consistent with the notion that the brain and kidney are more vulnerable to ischemia.

Unlike state-3 respiration, state-4 respiration did not change significantly following $30 \mathrm{~min}$ CA except for the moderate decrease (20\%) found in kidney mitochondria (Table 1). However, the activity in kidney mitochondria returned to the control rate following $45 \mathrm{~min}$ CA. State- 4 respiration of brain and liver mitochondria did not change following CA for $30 \mathrm{~min}$ or $45 \mathrm{~min}$. In heart mitochondria, the activity was increased by $70 \%$ only following $45 \mathrm{~min}$ CA. Overall, state- 4 respiration did not show a correlation with ischemic time and was not a good marker of ischemia.

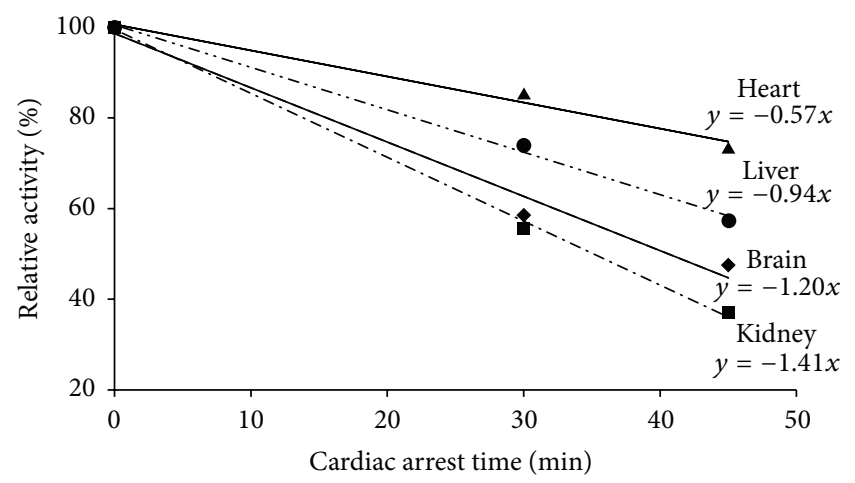

FIgURE 2: The decrease rates in state-3 respiration of heart, liver, brain, and kidney mitochondria following CA.

3.4. State-3 Respiration following CPB Resuscitation. We now focus on how state- 3 respiration observed following $30 \mathrm{~min}$ CA further changes following $60 \mathrm{~min}$ CPB resuscitation. Figure 3 shows that the average state- 3 respiration rate remained the same in brain mitochondria (Figure 3(a)). However, the average had a great variability; the activities of mitochondria from two rats ware recovered to the lower control range, whereas the activity of one animal decreased to $20 \%$ of the control rate. Since the average mitochondrial isolation yield also had a high variability, we plotted state3 respiration activity against isolation yield and found a positive correlation between them.

State-3 respiration of heart mitochondria was not changed following CPB resuscitation (Figure 3(b)). Interestingly, state-3 respiration of kidney and liver mitochondria was significantly recovered following CPB resuscitation. Kidney mitochondrial activity was increased to $70 \%$ of the control level (Figure 3(c)). Liver mitochondrial activity was completely recovered to the control level following $60 \mathrm{~min}$ CPB resuscitation (Figure $3(d)$ ).

\section{Discussion}

4.1. Mitochondrial Respiration in Ischemia and Reperfusion. Mitochondrial respiration is the central mechanism for ischemia [18-20] and normalizing mitochondrial respiration 


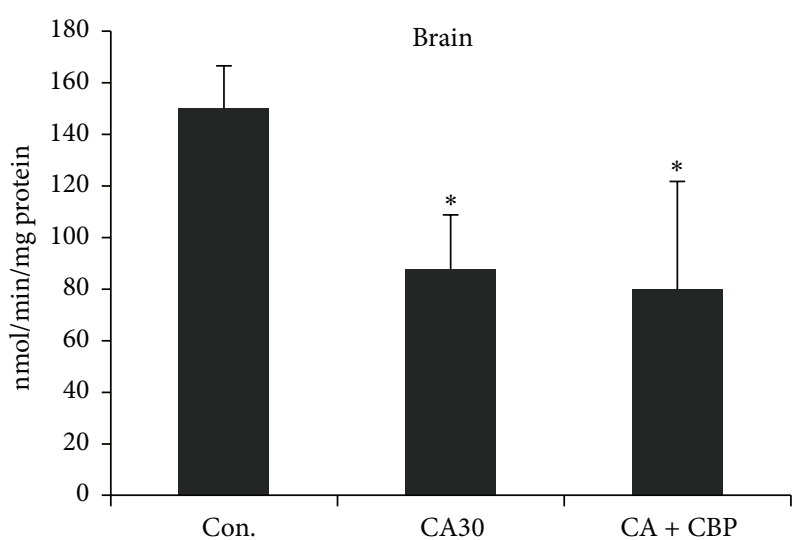

(a)

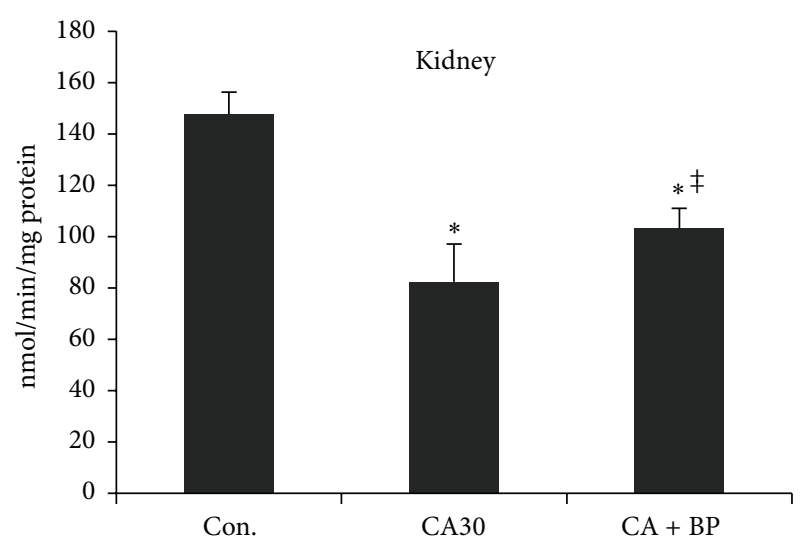

(c)

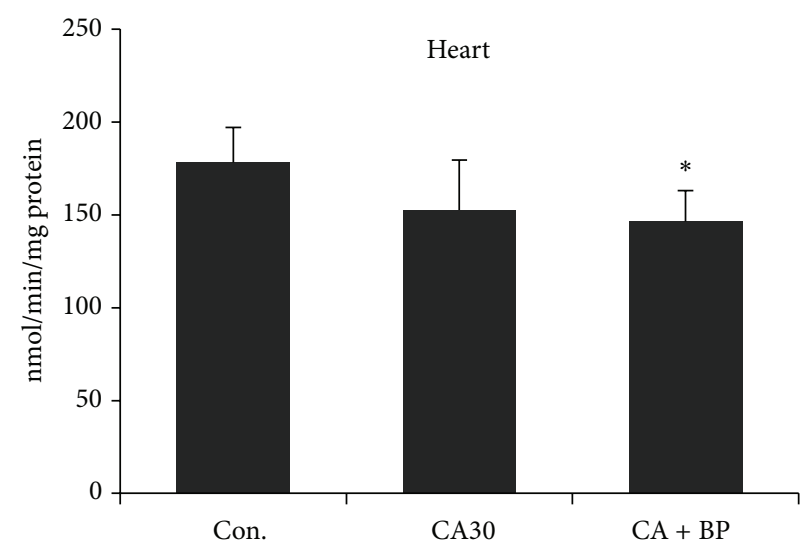

(b)

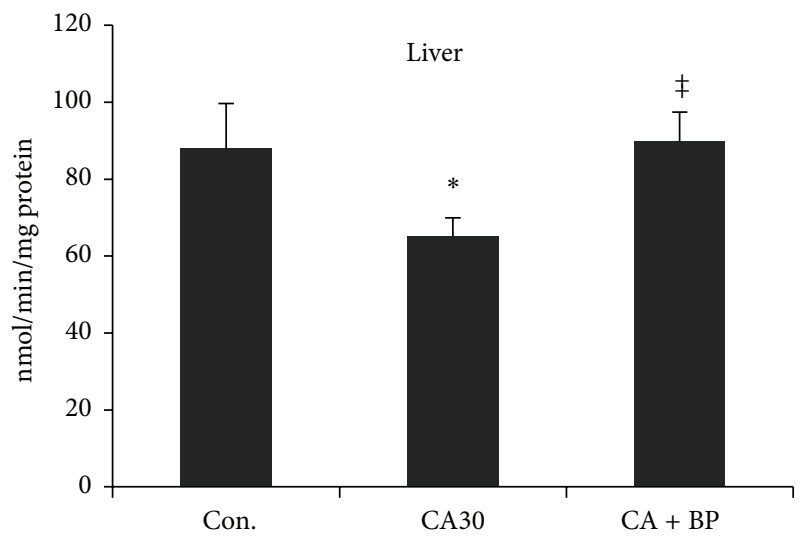

(d)

FIGURE 3: Changes in state-3 respiration activity of mitochondria following $30 \mathrm{~min}$ CA or $30 \mathrm{~min}$ CA and 60 min CPB resuscitation from brain, heart, kidney, and liver ( ${ }^{*}$ against control; ${ }^{\ddagger}$ against CA30).

is essential for the recovery of organ function as well as successful resuscitation $[9,21]$. Particularly, the linear response of the decrease in state- 3 respiration to the duration of ischemic time in all four organs (Figure 2) suggests that state3 respiration is a good indicator of the progression of ischemic damage in each tissue. Therefore, following the activity of state- 3 respiration will provide information on the efficacy of resuscitation protocols for each organ.

Another key observation is that mitochondrial isolation yield was not affected by the duration of ischemia. Mitochondrial isolation yields provide information on mitochondrial structural integrity. Any injury models, which cause morphological change, may induce changes in mitochondrial density. These mitochondria will be excluded during the isolation process, resulting in lower isolation yields. In this light, constant mitochondria isolation yields following $45 \mathrm{~min}$ CA confirm that ischemia does not cause significant changes in mitochondrial density and that there is no selective loss of damaged mitochondria.

4.2. Effect of Reperfusion on State-3 Respiration of Brain Mitochondria. The state-3 respiration activities of brain mitochondria from individual animals vary widely following 60 min $\mathrm{CPB}$ resuscitation (Figure 4); the activity in one animal recovers to $90 \%$, while in another animal it drops to $20 \%$ of the control activity. In addition, the isolation yields of brain mitochondria from most animals decrease with a high variability. The positive correlation between the activity and the yield (Figure 4) suggests that animals with more activity loss also suffer more from structural alterations of brain mitochondria. Therefore, it seems that activity loss is accompanied by structural alterations following resuscitation and proper perfusion can significantly recover the respiration activity with minimal structural alterations in mitochondria.

The decrease in isolation yield excludes no-reflow phenomenon as the reason for the loss of activity, because $90 \mathrm{~min}$ CA does not result in a decreased isolation yield (data not shown). Rather, it suggests that the inconsistent and/or insufficient perfusion is responsible for the inconsistent response of brain mitochondria to resuscitation. In support of this view, previous reports proposed that inadequate perfusion is more harmful than no perfusion [22]. The brain is a heterogeneous tissue with many compartments. A subtle difference in the severity of damage, microvascular obstruction, or edema may interfere with the blood flow to the brain resulting in inconsistent global or local perfusion. Mitochondria in regions with insufficient flow undergo additional damage accompanied by structural alterations. 


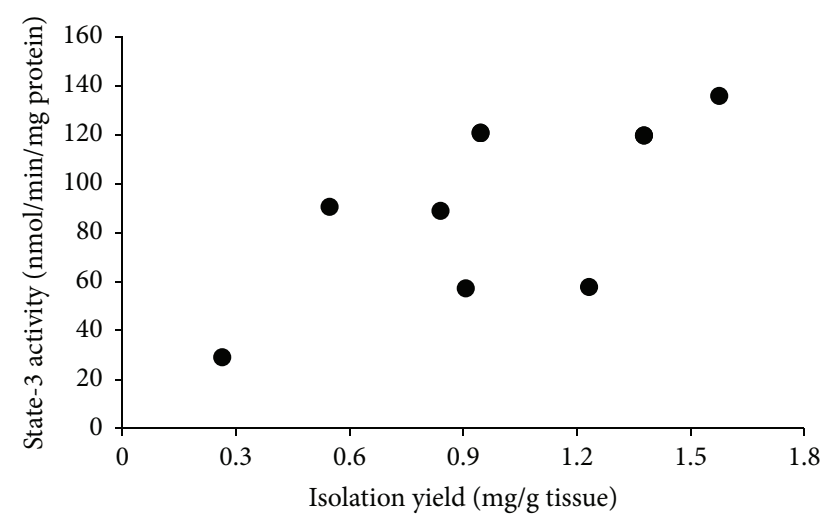

FIGURE 4: Dot plot of state-3 respiration against isolation yield of brain mitochondria following $30 \mathrm{~min} \mathrm{CA}$ and $60 \mathrm{~min} \mathrm{CPB}$ resuscitation.

Another possible explanation may be the existence of a damage threshold. Mitochondria damaged beyond the threshold (irreversible damage) during ischemia are subjected to additional activity loss and/or decomposition during reperfusion. On the other hand, mitochondria with slight damage recover with reperfusion, and, by $30 \mathrm{~min} \mathrm{CA}$, mitochondria with both reversible and irreversible damage can be observed in the brain. Overall, the high variability in respiration activity and isolation yield well represent the difficulty and complexity commonly observed in investigating brain perfusion in human patients and animal models [23].

\subsection{Effect of Reperfusion on State-3 Respiration of Heart} Mitochondria. The achievement of ROSC and hemodynamic data show the resumption of heart function following $\mathrm{CPB}$ resuscitation. However, the decrease in HR, MRP, and PP also showed that heart function was compromised at some degree (Figure 1). Consistent with this physiological outcome of the heart, the $15 \%$ decrease in state- 3 respiration, which occurred following CA, still exists following resuscitation. Interestingly, heart mitochondria with the least activity loss following CA do not show any sign of recovery following CPB resuscitation.

Preventing additional decreases in state- 3 respiration indicates that reperfusion significantly normalizes preceding ischemic alterations in the heart. However, this normalization is not sufficient to improve state- 3 respiration. The reason that mitochondrial respiration remained inhibited is not clear. One possible explanation is that reperfusion may have caused additional damage to mitochondria, as evidenced by the decrease in uncoupled respiration following $60 \mathrm{~min}$ resuscitation (Table 1 ). In any case, simply restoring blood flow will not completely rescue the heart from prolonged ischemia and additional intervention is required.

4.4. Effect of Reperfusion on State-3 Respiration of Kidney and Liver Mitochondria. The respiration activities of kidney and liver mitochondria are significantly recovered by CPB resuscitation, suggesting that sufficient perfusion substantially normalizes molecular alterations and metabolic disorders. It is noteworthy that kidney mitochondria lose state-3 activity more than the other mitochondria but still respond well to $\mathrm{CPB}$ resuscitation. The remaining 30\% inhibition of state- 3 respiration again may be caused by permanent ischemic damage or ongoing reperfusion injury. The remained increased tissue weight, which occurred following CA, suggests that ischemic alterations still exist at some degree and contribute to the $30 \%$ decrease in activity.

Following $60 \mathrm{~min} \mathrm{CPB}$ resuscitation, state- 3 respiration of liver mitochondria is completely recovered to control level. Mitochondrial isolation yield also becomes normal. The recovery of liver seems to be the most prominent of the four organs by $\mathrm{CPB}$ resuscitation. Apparently, no reperfusion injury is evident in the state- 3 respiration in liver mitochondria following $60 \mathrm{~min} \mathrm{CPB}$ resuscitation. The results suggest that the liver is the most resistant to ischemia/reperfusion injury.

Overall, the results show that following state-3 respiration is a useful approach to gain insights into how each tissue responds to ischemia and resuscitation. Time course studies varying the duration of $\mathrm{CA}$ and resuscitation will help to better understand how injury severity affects the response of each organ to different resuscitation protocols. In addition, correlation of state- 3 respiration with the physiological outcome and survival of animals following resuscitation will elucidate the role of mitochondrial respiration for ischemia/reperfusion injury in CA.

\section{Conclusion}

Using $30 \mathrm{~min}$ CA and 60 min CPB resuscitation, we study the effect of ischemia and reperfusion on mitochondrial respiration in the brain, heart, kidney, and liver. State-3 respiration of mitochondria from all four organs decreases proportionally to the duration of ischemic time. The decrease rates are greater in brain and kidney mitochondria than in heart and liver mitochondria. Following $60 \mathrm{~min}$ CPB resuscitation, state-3 respiration activity of brain mitochondria varies from animal to animal. This inconsistent response of brain mitochondria well represents the difficulty in studying the response of brain to resuscitation in human patients and animal models. The respiration activity does not change in heart mitochondria and significantly increases in kidney and liver mitochondria. The results suggest that each organ responds differently to $\mathrm{CPB}$ resuscitation and this difference should be considered to design better resuscitation protocols.

\section{Conflict of Interests}

The authors declare that there is no conflict of interests regarding the publication of this paper.

\section{Acknowledgment}

This work was supported by the NIH grant (RO1 HL067630). 


\section{References}

[1] B. W. Roberts, J. H. Kilgannon, M. E. Chansky et al., "Multiple organ dysfunction after return of spontaneous circulation in postcardiac arrest syndrome," Critical Care Medicine, vol. 41, no. 6, pp. 1492-1501, 2013.

[2] H. Li, L. Zhang, Z. Yang et al., "Even four minutes of poor quality of CPR compromises outcome in a porcine model of prolonged cardiac arrest," BioMed Research International, vol. 2013, Article ID 171862, 6 pages, 2013.

[3] H. Jiang, F. Meng, W. Li, L. Tong, H. Qiao, and X. Sun, "Splenectomy ameliorates acute multiple organ damage induced by liver warm ischemia reperfusion in rats," Surgery, vol. 141, no. 1, pp. 32-40, 2007.

[4] L. W. Czerski, P. A. Szweda, and L. I. Szweda, "Dissociation of cytochrome $\mathrm{c}$ from the inner mitochondrial membrane during cardiac ischemia," Journal of Biological Chemistry, vol. 278, no. 36, pp. 34499-34504, 2003.

[5] Q. Chen, S. Moghaddas, C. L. Hoppel, and E. J. Lesnefsky, "Ischemic defects in the electron transport chain increase the production of reactive oxygen species from isolated rat heart mitochondria," American Journal of Physiology-Cell Physiology, vol. 294, no. 2, pp. C460-C466, 2008.

[6] S. Rehncrona, L. Mela, and B. K. Siesjo, "Recovery of brain mitochondrial function in the rat after complete and incomplete cerebral ischemia," Stroke, vol. 10, no. 4, pp. 437-446, 1979.

[7] M. Iwase, "The influence of portal vein occlusion on liver mitochondria in rats after releasing biliary obstruction," Nagoya Journal of Medical Science, vol. 48, no. 1-4, pp. 11-20, 1986.

[8] B. Gonzalez-Flecha, J. C. Cutrin, and A. Boveris, "Time course and mechanism of oxidative stress and tissue damage in rat liver subjected to in vivo ischemia-reperfusion," Journal of Clinical Investigation, vol. 91, no. 2, pp. 456-464, 1993.

[9] J. Jiang, X. Fang, Y. Fu, W. Xu, L. Jiang, and Z. Huang, "Impaired cerebral mitochondrial oxidative phosphorylation function in a rat model of ventricular fibrillation and cardiopulmonary resuscitation," BioMed Research International, vol. 2014, Article ID 192769, 9 pages, 2014.

[10] R. W. Neumar, N. G. Bircher, K. M. Sim et al., "Epinephrine and sodium bicarbonate during CPR following asphyxial cardiac arrest in rats," Resuscitation, vol. 29, no. 3, pp. 249-263, 1995.

[11] P. K. E. W. Ballaux, T. Gourlay, C. P. Ratnatunga, and K. M. Taylor, "A literature review of cardiopulmonary bypass models for rats," Perfusion, vol. 14, no. 6, pp. 411-417, 1999.

[12] F. Han, M. Boller, W. Guo et al., "A rodent model of emergency cardiopulmonary bypass resuscitation with different temperatures after asphyxial cardiac arrest," Resuscitation, vol. 81, no. 1, pp. 93-99, 2010.

[13] H. R. Scholte, Y. Yu, J. D. Ross, I. I. Oosterkamp, A. M. C. Boonman, and H. F. M. Busch, "Rapid isolation of muscle and heart mitochondria, the lability of oxidative phosphorylation and attempts to stabilize the process in vitro by taurine, carnitine and other compounds," Molecular and Cellular Biochemistry, vol. 174, no. 1-2, pp. 61-66, 1997.

[14] S. Krahenbuhl, M. Chang, E. P. Brass, and C. L. Hoppel, "Decreased activities of ubiquinol:ferricytochrome c oxidoreductase (complex III) and ferrocytochrome c:oxygen oxidoreductase (complex IV) in liver mitochondria from rats with hydroxycobalamin[c-lactam]-induced methylmalonic aciduria," The Journal of Biological Chemistry, vol. 266, no. 31, pp. 20998-21003, 1991.
[15] J. Kim, H. Fujioka, N. L. Oleinick, and V. E. Anderson, "Photosensitization of intact heart mitochondria by the phthalocyanine Pc4: correlation of structural and functional deficits with cytochrome c release," Free Radical Biology and Medicine, vol. 49, no. 5, pp. 726-732, 2010.

[16] J. Kim, T. Yin, M. Yin et al., "Examination of physiological function and biochemical disorders in a rat model of prolonged asphyxia-induced cardiac arrest followed by cardio pulmonary bypass resuscitation," PLOS ONE, vol. 9, no. 11, Article ID el12012, 2014.

[17] J. Kim, J. Lampe, T. Yin, K. Shinozaki, and L. Becker, "Phospholipid alterations in the brain and heart in a rat model of asphyxia-induced cardiac arrest and cardiopulmonary bypass resuscitation," Molecular and Cellular Biochemistry, 2015.

[18] V. Borutaite, A. Toleikis, and G. C. Brown, "In the eye of the storm: mitochondrial damage during heart and brain ischaemia," The FEBS Journal, vol. 280, no. 20, pp. 4999-5014, 2013.

[19] N. R. Sims and H. Muyderman, "Mitochondria, oxidative metabolism and cell death in stroke," Biochimica et Biophysica Acta, vol. 1802, no. 1, pp. 80-91, 2010.

[20] C. Dezfulian, S. Shiva, A. Alekseyenko et al., "Nitrite therapy after cardiac arrest reduces reactive oxygen species generation, improves cardiac and neurological function, and enhances survival via reversible inhibition of mitochondrial complex I," Circulation, vol. 120, no. 10, pp. 897-905, 2009.

[21] S. T. Yeh, H.-L. Lee, S. E. Aune, C.-L. Chen, Y.-R. Chen, and M. G. Angelos, "Preservation of mitochondrial function with cardiopulmonary resuscitation in prolonged cardiac arrest in rats," Journal of Molecular and Cellular Cardiology, vol. 47, no. 6, pp. 789-797, 2009.

[22] B. C. White, J. F. Hildebrandt, A. T. Evans et al., "Prolonged cardiac arrest and resuscitation in dogs: brain mitochondrial function with different artificial perfusion methods," Annals of Emergency Medicine, vol. 14, no. 5, pp. 383-388, 1985.

[23] J. Pan, A.-A. Konstas, B. Bateman, G. A. Ortolano, and J. Pile-Spellman, "Reperfusion injury following cerebral ischemia: pathophysiology, MR imaging, and potential therapies," Neuroradiology, vol. 49, no. 2, pp. 93-102, 2007. 


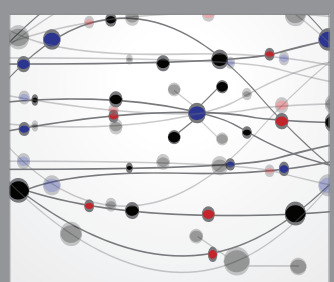

The Scientific World Journal
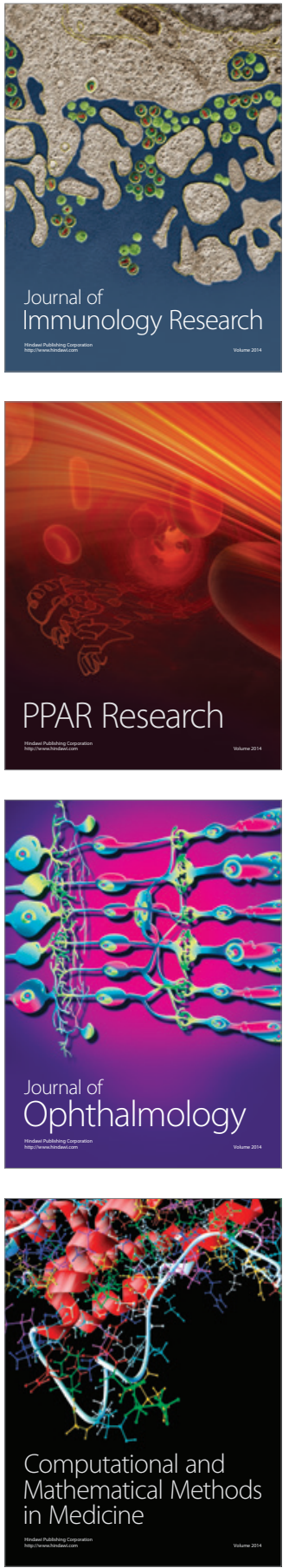

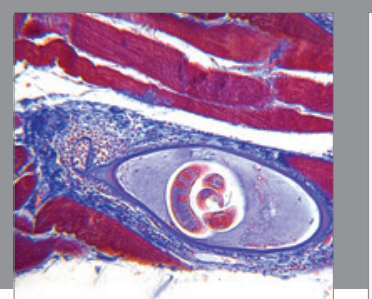

Gastroenterology Research and Practice

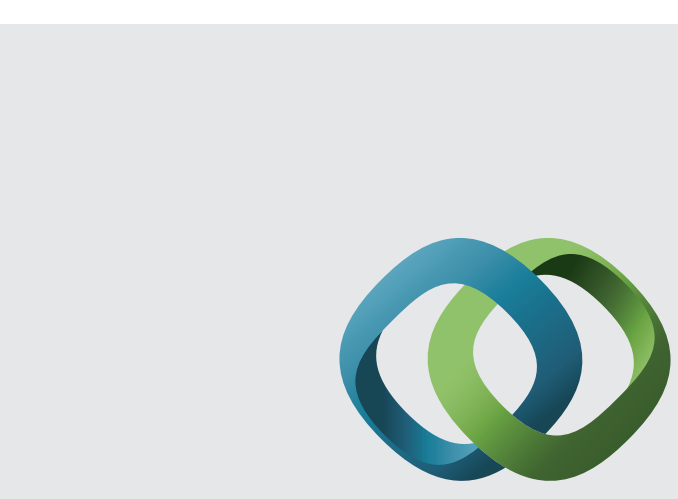

\section{Hindawi}

Submit your manuscripts at

http://www.hindawi.com
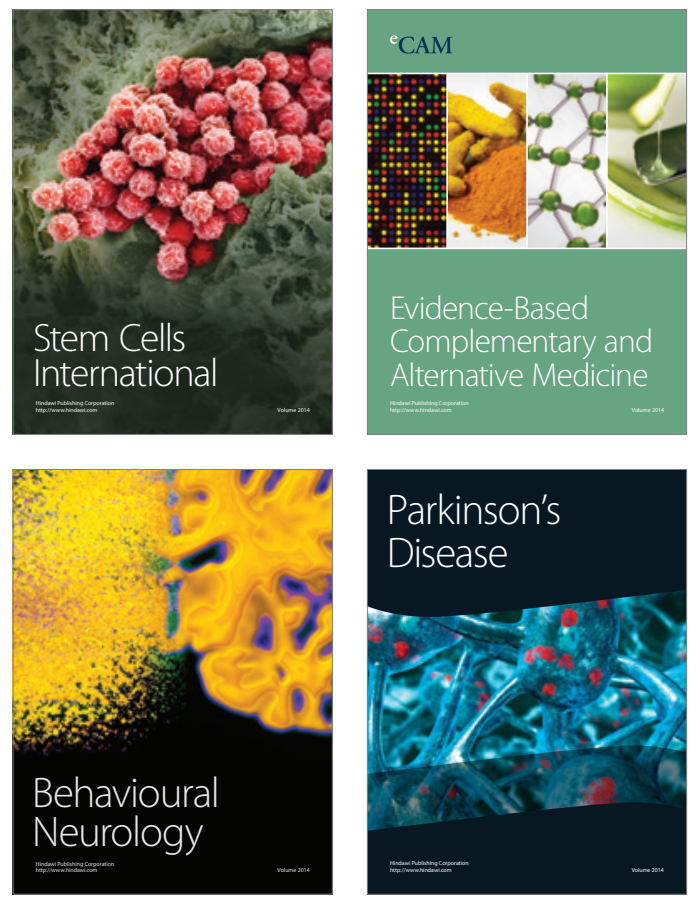
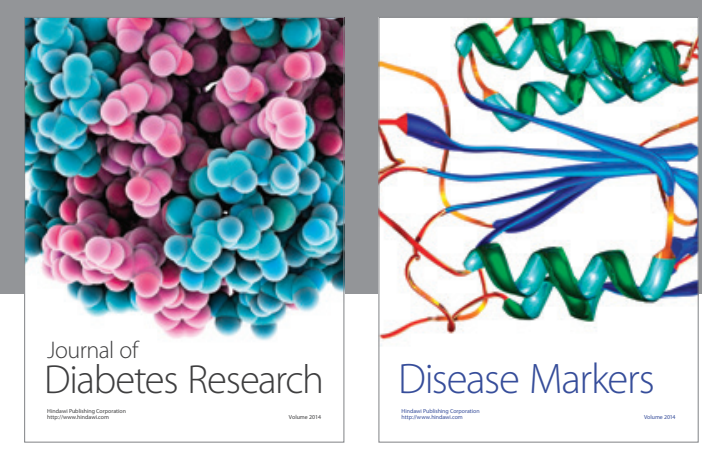

Disease Markers
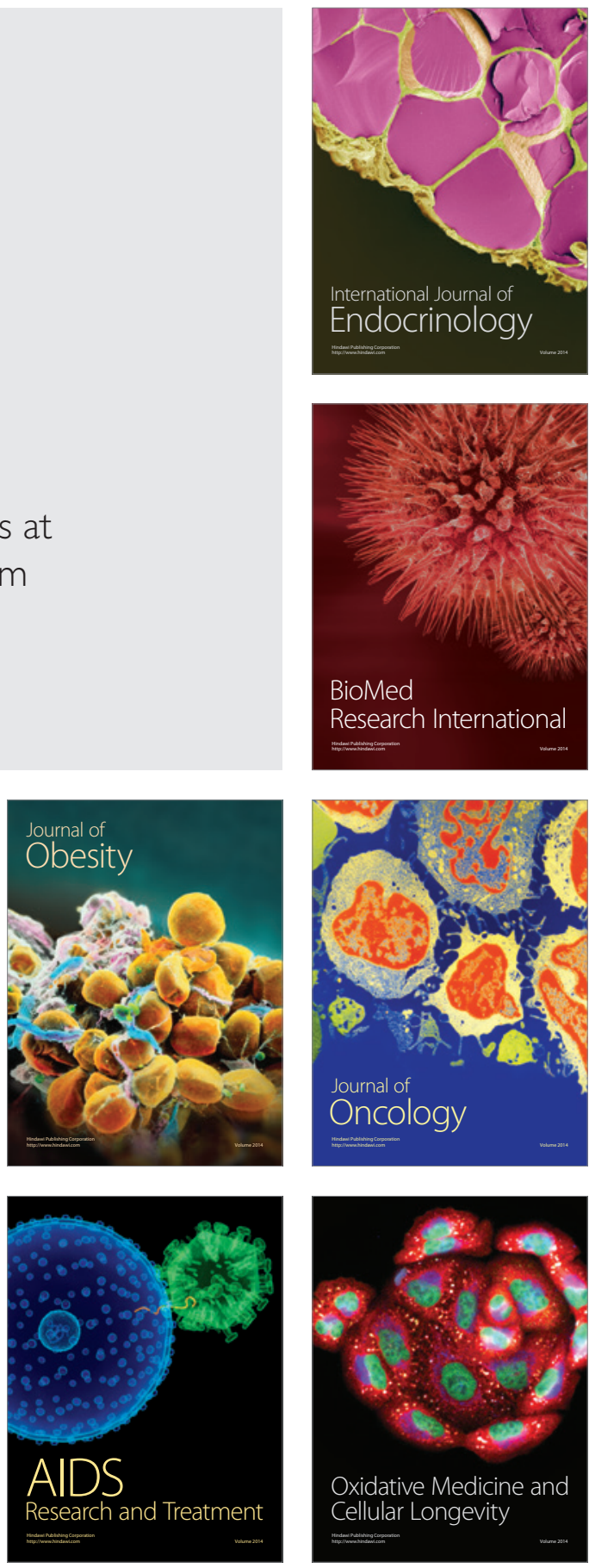